\title{
Vaccination can drive an increase in frequencies of antibiotic resistance among nonvaccine serotypes of Streptococcus pneumoniae
}

\author{
Uri Obolski ${ }^{a, 1}$, José Lourenço ${ }^{a}$, Craig Thompson ${ }^{a}$, Robin Thompson ${ }^{a}$, Andrea Gori ${ }^{b}$, and Sunetra Gupta ${ }^{a}$

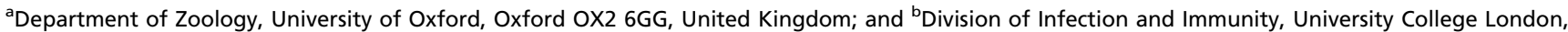 \\ London WC1E 6BT, United Kingdom
}

Edited by Bruce R. Levin, Emory University, Atlanta, GA, and approved February 13, 2018 (received for review October 26, 2017)

\begin{abstract}
The bacterial pathogen Streptococcus pneumoniae is a major public health concern, being responsible for more than 1.5 million deaths annually through pneumonia, meningitis, and septicemia. Available vaccines target only a subset of serotypes, so vaccination is often accompanied by a rise in the frequency of nonvaccine serotypes. Epidemiological studies suggest that such a change in serotype frequencies is often coupled with an increase of antibiotic resistance among nonvaccine serotypes. Building on previous multilocus models for bacterial pathogen population structure, we have developed a theoretical framework incorporating variation of serotype and antibiotic resistance to examine how their associations may be affected by vaccination. Using this framework, we find that vaccination can result in a rapid increase in the frequency of preexisting resistant variants of nonvaccine serotypes due to the removal of competition from vaccine serotypes.
\end{abstract}

mathematical model | antibiotic resistance | epidemiology | ecological competition | Streptococcus pneumoniae

$\mathbf{T}^{\mathrm{h}}$ he bacterial pathogen Streptococcus pneumoniae (otherwise known as pneumococcus) is estimated to be responsible for one-third of all pneumonia cases, annually causing tens of millions of severe infections worldwide (1). Two major tools are available to combat pneumococcal disease: antibiotic treatment and vaccination (2). Unfortunately, the effectiveness of antibiotic drugs is often impaired by the emergence and spread of antibiotic-resistant pneumococci (3). Antibiotic-resistant pneumococcal infections annually account for tens of thousands additional hospitalizations and outpatient visits in the United States alone, resulting in $\sim 230$ million US dollars of additional costs (4). Furthermore, antibiotic-resistant pneumococcal infections increase the length of patient hospitalizations (5) and cause treatment failures (6, $7)$. Although it is difficult to determine the effects of antibiotic resistance on clinical outcomes (7), a number of studies have reported a significant increase in pneumococcal pneumonia mortality due to penicillin resistance (7-10). In contrast, vaccination can prevent new pneumococcal infections by increasing host immunity against pneumococcal serotypes. However, available vaccines, such as the pneumococcal conjugate vaccine (PCV7 and PCV 13), target only a subset of the more than 90 circulating pneumococcal serotypes (11). The selective pressure exerted by such vaccines can increase frequencies of nonvaccine serotypes, a phenomenon termed vaccineinduced serotype replacement (12-14). Changes in the broader genetic composition of pneumococci have also been observed after vaccination $(15,16)$, and it has been proposed that vaccination may induce a shift in the metabolic profiles of nonvaccine strains (known as vaccine-induced metabolic shift, or VIMS), as a consequence of resource competition among bacteria sharing the same metabolic niches $(17,18)$.

The deployment of pneumococcal vaccines has also led to significant changes in the frequencies of antibiotic resistance of pneumococci. As might be expected, vaccines targeting pneumococcal serotypes that have high initial resistance frequencies have led to the reduction of resistance at the population level in certain settings (19-23). However, it is not easy to account for a postvaccination increase in resistance frequencies of subsets of nonvaccine type (nVT) pneumococci, as has been repeatedly observed in a range of locations (24-32). Fig. $1 A$ shows changes in antibiotic resistance within the 19A serotype, an nVT of PCV7, following the introduction of this vaccine in 2000 within pneumococcal isolates of children $\leq 5 \mathrm{y}$ of age, collected in Massachusetts (16); there was an increase in the minimum inhibitory concentration (MIC) of erythromycin between 2001 and 2007, whereas resistance to penicillin and ceftriaxone remained unchanged (Fig. 1A). It should be noted that the increase in erythromycin resistance occurred despite macrolide prescription rates not increasing in the population over the study period (33, 34). A Bayesian analysis of population structure (BAPS) revealed a significant admixture of antibiotic resistance-associated alleles from various serotypes (Fig. 1B; see Methods for a full description). This analysis demonstrated that only $87 \%$ of antibiotic resistanceassociated allele combinations within serotype 19A were found to have originated from the 19A serotype itself, with the rest much more likely to have originated in other serotypes. Together with the lack of increase in antibiotic prescription in the study population, this indicates that 19A strains might be responding to changing selective pressures arising from the pneumococcal population structure postvaccination. The analysis was repeated for another publicly available UK pneumococcal dataset (35) in which the 19A serotype had the second-highest value of

\section{Significance}

Vaccination is an important tool for decreasing the spread and disease burden of the widespread bacterial pathogen Streptococcus pneumoniae. However, current vaccines target only a subset of pneumococcal serotypes, and the nonvaccine types often remain at high frequencies after vaccination. In some cases, nonvaccine types display a disconcerting increase in antibiotic resistance frequencies after a population has been vaccinated by the commonly used PCV7 or PCV13, and the reason for this is unclear. Using mathematical modeling, we show that removal of competition with the vaccine types may induce this rise in antibiotic resistance. Our model explores the conditions leading to such an increase and contributes to a better understanding of this process.

Author contributions: U.O., J.L., and S.G. designed research; U.O. and S.G. performed research; U.O., J.L., C.T., R.T., A.G., and S.G. analyzed data; and U.O., J.L., C.T., R.T. A.G., and S.G. wrote the paper.

The authors declare no conflict of interest.

This article is a PNAS Direct Submission.

Published under the PNAS license.

${ }^{1}$ To whom correspondence should be addressed. Email: uriobolski@gmail.com.

This article contains supporting information online at www.pnas.org/lookup/suppl/doi:10 1073/pnas.1718712115/-/DCSupplemental.

Published online March 6, 2018. 

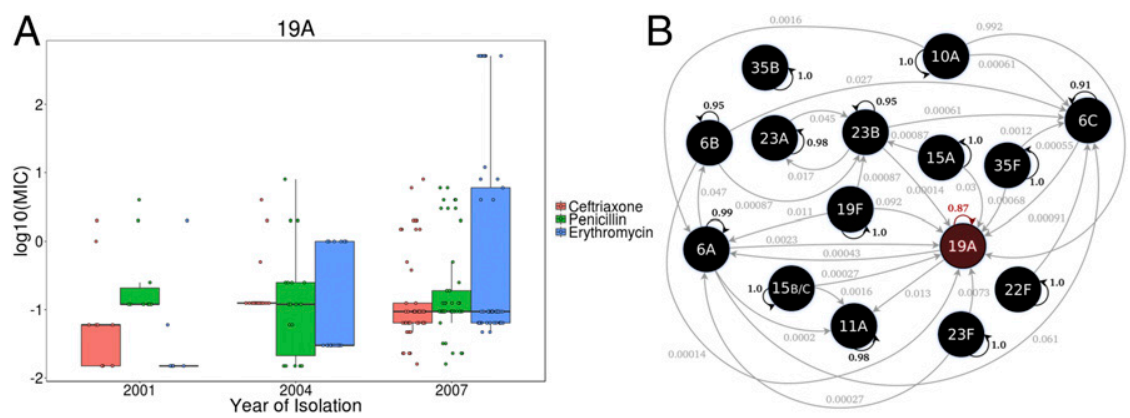

Fig. 1. Observed changes in resistance, and admixture estimation of the $19 \mathrm{~A}$ serotype. $(A)$ Box plots of $\log _{10}(\mathrm{MIC})$ of different antibiotics, with actual values overlaid as points. Serotype 19A increases in MIC of erythromycin ( $P$ value $<2 \cdot 10^{-7}$, Spearman correlation $\left.=0.58\right)$ but not of penicillin and ceftriaxone $(P$ value $=0.56,0.57$; Spearman correlation $=-0.07$, both), between 2001 and 2007 after PCV7 introduction at 2000. (B) Admixture analysis using BAPS (see Methods) on antibiotic resistance-associated genes on the same data as in $A$. Arrows represent statistically significant admixture events $(P$ value for each event $<0.001$ ), with arrow directions defining origin and destination of admixed alleles, and numbers representing the fraction of alleles contributed from source to receiving serotype. Serotype 19A has the highest fraction of alleles most likely from other serotypes, with only $87 \%$ of alleles likely to originate from serotype 19A's population.

antibiotic resistance alleles which most likely originate from other serotypes (SI Appendix, Fig. S1).

Here, we explore the interactive evolution of vaccination and antibiotic resistance using a multilocus model of serotype and antibiotic resistance with full flexibility in associations between alleles, reflecting the high levels of admixture outlined above. We assume that there is a cost to resistance, and, consequently, a resistant strain has a reduced chance of cocolonizing individuals already colonized with a susceptible strain. Thus, in contrast with previous multilocus models of pneumococcal evolution $(17,18)$ that assume interference occurs between organisms carrying similar metabolic and virulence alleles, our model assumes that stronger interference occurs between strains with different antibiotic resistance profiles. We find that a postvaccination surge in antibiotic resistance frequencies can occur in nVTs under these circumstances and, furthermore, may be hastened by asymmetries in the rate of acquisition of resistance to different antibiotics.

\section{Results}

Model Structure. We investigate the impact of vaccination within a system containing two pneumococcal serotypes, $a$ and $b$, of which the first is included in the vaccine (VT) and the second is not (nVT), under selection pressure from two antibiotics, $\mathrm{X}$ and $\mathrm{Y}$. We assume that immunity is serotype-specific but may be incomplete, with its efficacy represented in our model by the parameter $0 \leq \gamma \leq 1$, where $\gamma=1$ implies that immunity is complete, and $\gamma=0$ corresponds to no serotype-specific immunity. Our model allows for cocolonization by two pneumococcal strains, and, when $\gamma<1$, the cocolonizing strains can belong to the same serotype. We denote a bacterial strain of serotype $a$ or $b$ as having a resistance profile $j$, where $j$ takes values in $\{00,01,10\}$, corresponding to sensitivity to both antibiotics, resistance to antibiotic $X$, and resistance to antibiotic $Y$, respectively.

The intrinsic transmissibility of a strain can be represented by its basic reproductive number (36), $R_{0}$, which is a product of the duration of colonization $(D)$ and degree of infectivity $(\beta)$, where the latter is, effectively, a combination of parameters defining the likelihood of acquisition by a susceptible individual of a particular strain from a colonized individual. We assume that the fitness cost of antibiotic resistance [for which estimates vary substantially for pneumococci (37-40), as well as for other bacterial species $(38,41-43)$ ] can translate into lower infectivity of resistant strains compared with sensitive strains $\left(\beta_{00}>\beta_{01}\right)$. However, the duration of carriage may be longer for resistant strains (44) due to antibiotic usage $\left(D_{01}>D_{00}\right)$. Consequently, in the absence of antibiotic usage, the basic reproductive number of resistant strains, $R^{01}{ }_{0}$, will typically be lower than the basic reproductive number of sensitive strains, $R^{00}{ }_{0}$, but this can be reversed with antibiotic usage. High serotype-specific immunity leads to the competitive exclusion of the strain with the lower $R_{0}$ within a given serotype (35) Therefore, in the absence of antibiotics, the susceptible strain would dominate, but an increase in antibiotic usage could reverse this outcome. Lower levels of serotype-specific immunity can lead to the coexistence of resistant and susceptible strains within a serotype, and an increase in antibiotic usage could either cause an increase in the resistance frequencies or lead to the exclusion of the susceptible strain. These properties of our model are identical to previous work focusing on single serotypes (45-47).

Introducing more serotypes does not alter the outcome of competition between resistant and susceptible strains within a given serotype unless there is some form of competitive interaction between serotypes. This may be mediated by serotypetranscending immune responses or by ecological competition. Here, we incorporate ecological competition by introducing a parameter $(0 \leq \psi \leq 1)$ which captures the degree to which intrinsic fitness differences (such as in growth rates) between resistant and susceptible strains may allow an individual carrying a susceptible strain of pneumococci to suppress cocolonization by a resistant strain. This could be thought of as a form of preemptive competition, where susceptible bacteria in a colonized individual would preclude the introduction of a resistant strain as they start with greater numbers and have a fitness advantage. We emphasize that this is a form of ecological competition between bacterial strains and is not mediated by immunity; thus, individuals carrying strain $a_{00}$ may not be available for cocolonization by either $b_{10}$ or $b_{01}$ (for example, if $\psi=1$ ) but will be fully susceptible to further colonization by $b_{00}$.

Vaccination Can Increase the Frequency of Antibiotic Resistance Among nVTs. We start by considering a model in which the two serotypes, $a$ and $b$, are either susceptible or resistant to a single antibiotic (Y) and there are no serotype-specific differences in $R_{0}$. The equilibrium frequencies of the four strains $\left(a_{00}, a_{01}, b_{00}, b_{01}\right)$ before vaccination are determined by the degree of serotype-specific immunity $(\gamma)$ and inhibition of cocolonization by resistant strains $(\psi)$ within the system, as well as the basic reproductive numbers of sensitive and resistant strains $\left(R_{0}^{00}\right.$ and $R_{0}^{01}$, respectively).

High serotype-specific immunity leads to competitive exclusion within a given serotype, typically of the strain with the lower $R_{0}$ (45). However, the cost imposed on resistant strains through inhibition of cocolonization $(\psi>0)$ can lead to their exclusion even if they have a slightly higher $R_{0}$. The removal of serotype $a$ (the VT) through vaccination may cause a reversal of the 
outcome of competition between $b_{00}$ and $b_{01}$, with the resistant $\mathrm{nVT}$ completely replacing the susceptible nVT, as shown in Fig. $2 A$. This is due to the removal of ecological competition between $a_{00}$ and $b_{01}$, and can be observed under circumstances where $R^{01}{ }_{0}$ is somewhat in excess of $R_{00}^{00}$, provided $\psi$ is above the following threshold (see SI Appendix, Supplementary Information S2 for derivation):

$$
\psi>\frac{\frac{2 \gamma}{\mu D_{00}}\left(\frac{1}{R_{0}^{00}}-\frac{1}{R_{0}^{01}}\right)}{\left(1-\frac{1}{R_{0}^{00}}\right)\left(1-\left(\frac{1}{\gamma}\left(1-\frac{1}{R_{0}^{00}}\right)\right)\right)} .
$$

When serotype-specific immunity is complete $(\gamma=1)$, the region where a surge in frequency of the resistant nVT occurs is limited to the area between $R^{01}{ }_{0}>R^{00}{ }_{0}$ and the curve described by Eq. 1 . When $R^{01}{ }_{0}<R^{00}{ }_{0}$, there is no change in the outcome of withinserotype competition, as the susceptible nVT will continue to dominate after the VT is removed (Fig. $2 C$, left of the dashed line); when $\mathrm{R}^{01}{ }_{0}$ is sufficiently in excess of $\mathrm{R}^{00}{ }_{0}, b_{01}$ will have displaced $b_{0 O}$ before vaccination and no change will be seen (Fig. $2 C$, right of the solid black curve).

At lower levels of serotype-specific immunity $(\gamma<1)$, susceptible and resistant strains may coexist within the same serotype within certain boundaries of difference in $R_{0}(45-47)$. In this case, the resistant strain will increase in prevalence after vaccination, due to the cessation of competition from $a_{00}$, but may still remain the rarer strain (Fig. $2 B$ ). The range of differences in $R_{0}$ over which a surge in antibiotic resistance occurs is markedly wider, although the actual increase in frequency will be more limited (Fig. $2 D$ and $E$ ).

We next considered a scenario where serotypes differ in $R_{0}$, because of either unequal transmission coefficients or duration of carriage, both of which have been shown to differ substantially between serotypes (48). A 1.5 -fold increase in the $R_{0}$ of serotype $a$ leads to an expansion in the parameter range within which there is a postvaccination surge in $b_{01}$ (Fig. $2 F-H$; see a threefold increase leading to similar results in SI Appendix, Fig. $\mathrm{S} 2$ ). This is because the prevalence of $a_{00}$ increases, causing $b_{01}$ to be suppressed further. Consequently, $b_{01}$ experiences a greater increase in frequency when $a_{00}$ is removed by vaccination.

Importantly, the general principles illustrated above remain unaltered when we introduce a second antibiotic, $X$, with prevaccination equilibria falling into three categories: $(i)$ coexistence of all strains, (ii) competitive exclusion of both antibiotic-resistant strains $\left(b_{10}\right.$ and $\left.b_{01}\right)$, and (iii) competitive exclusion of the resistant strain with the lower $R_{0}$ (say $b_{10}$ ). Following the removal of serotype $a$ through vaccination, resistant strains that are already present will increase in frequency, and there may be an emergence of strains that were excluded before vaccination (SI Appendix, Fig. S3).

Effect of Asymmetries in Rates of Acquisition of Resistance to Different Antibiotics. The model can be extended to explicitly incorporate rates of resistance acquisition to two antibiotics, $\mathrm{X}$ and $\mathrm{Y}$, by introducing the parameter $\omega_{j}$ to describe the probability of a sensitive strain acquiring a resistance profile $j$ (see Methods). We find that this has a significant impact on the outcome of vaccination where the less transmissible strain (in this case, $b_{10}$, which is resistant to $\mathrm{X}$ ) is associated with a higher rate of resistance acquisition $\left(\omega_{01}<\omega_{10}\right)$. Under these circumstances, $b_{10}$ may stabilize at higher prevaccination frequencies than $b_{01}$, despite a significant transmissibility disadvantage (in Fig. $3 A, R^{10}{ }_{0}=0.91 R^{01}{ }_{0}$ ). The removal of competition from $a_{00}$ due to vaccination effectively unmasks the transmission advantage of $b_{01}$, thereby driving a rapid increase in its frequency. A stochastic implementation of this model indicates that a very significant rise in the frequency of $b_{01}$ can occur within a decade or two after the removal of the VT (Fig. $3 B$ ) under realistic parameter combinations, and that it has a strong likelihood of eventually displacing $b_{00}$ as the dominant strain within this serotype. Moreover, there is a high likelihood that the time point at which $b_{01}$ first becomes more common than $b_{10}$ is within a decade from the start of vaccination. This outcome is consistent

A
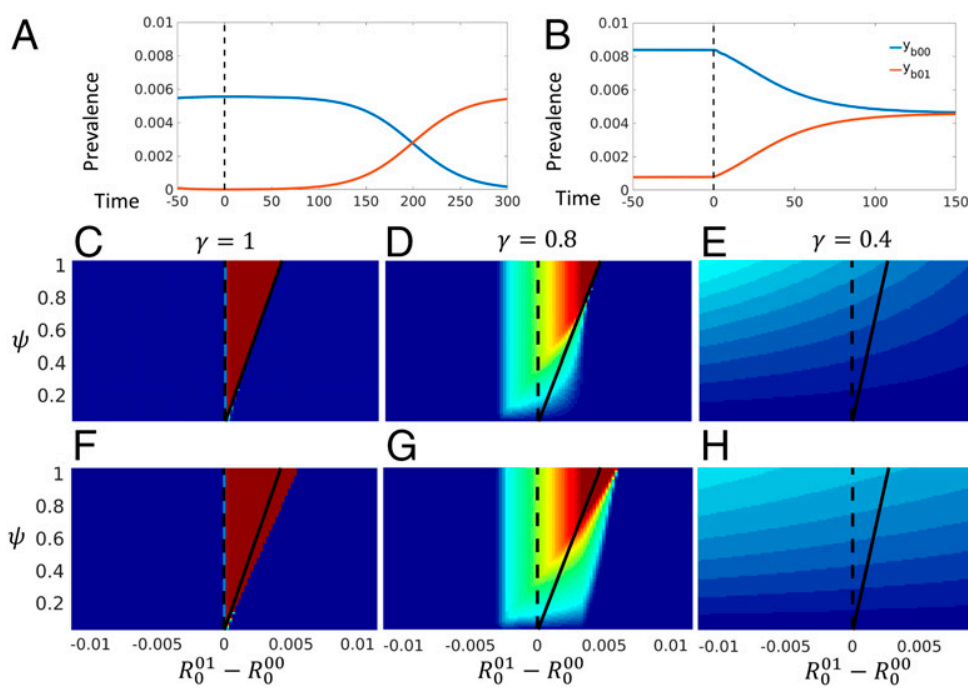

G
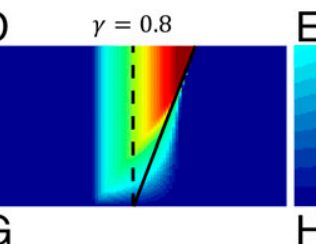

$\mathrm{E}$

$\mathrm{H}$
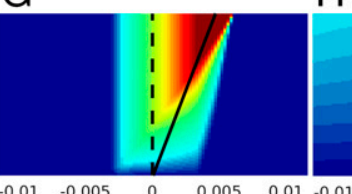

$R_{0}^{01}-R_{0}^{00}$

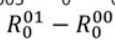

$0.01-0.01-0.005$ $R_{0}^{01}-R_{0}^{00}$

Fig. 2. Vaccination can increase the frequency of antibiotic resistance among nonvaccine serotypes. ( $A$ and $B$ ) Dynamics of susceptible nVT (blue), and resistant nVT (red) prevaccination and postvaccination (the time of which is marked by the dashed vertical line). ( $A$ ) Dynamics under full serotype-specific immunity $\left(\gamma=1, \psi=0.8 ; R^{01}{ }_{0}=1.504 ; R^{00}{ }_{0}=1.5\right)$. Prevaccination equilibrium values of VTs are 0 and 0.009, for $y_{a 01}$ and $y_{a 00}$, respectively. ( $B$ ) Dynamics under intermediate serotype-specific immunity $\left(\gamma=0.6, \psi=0.8 ; R^{01}{ }_{0}=R^{00}{ }_{0}=1.5\right)$. Prevaccination equilibrium values of VTs are 0.007 and 0.008 , for $y_{a 01}$ and $y_{a 00}$ respectively. ( $C-E)$ Heat maps of the increase in relative frequency of the resistant $\mathrm{nVT}\left(b_{01}\right)$ postvaccination against varying values of cocolonization inhibition $(\psi)$ and the difference in reproductive number between resistant and susceptible nVTs $\left(R^{01}{ }_{0}-R^{00}{ }_{0}\right.$, with $\left.R^{00}{ }_{0}=1.5\right)$. The black curves are derived from Eq. 1, and they mark the parameter ranges under which $b_{01}$ switches from being the rare to the common type postvaccination. The dashed vertical lines mark where $\mathrm{R}^{01}{ }_{0}=\mathrm{R}^{00}{ }_{0}$. $(F-H)$ VTs are assigned an increased reproduction number, expanding the range of parameters where a surge in resistance frequencies occurs; the curves obtained from Eq. 1 have been overlaid for ease of comparison with $C-E$. For $A, B$, and $F-H$, the transmission of the VTs was increased to 1.5 fold of the nVTs' reproductive number. 

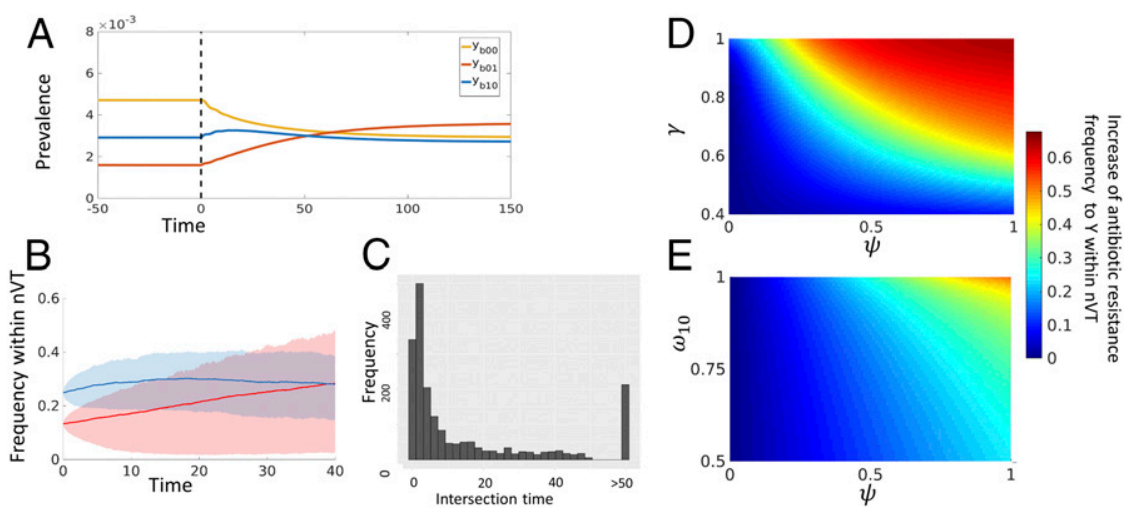

Fig. 3. An increase in antibiotic resistance under asymmetric acquisition rates. (A) Dynamics of nVT prevaccination and postvaccination, the time of which is marked by the dashed vertical line. We assume acquisition of resistance to $X\left(b_{00} \rightarrow b_{10}\right)$ is higher than to $Y\left(b_{00} \rightarrow b_{01}\right)$, but that $b_{10}$ is less transmissible than $b_{01}$. Prevaccination equilibrium values of VTs are $0.0055,0.0054$, and 0.0048 , for $y_{a 00}, y_{a 01}$, and $y_{a 10}$, respectively. (B) Stochastic implementation of the postvaccination dynamics (corresponding to the dynamics in $A$ ). Average values of 200 simulations are given by solid lines; interquartile range of simulation results is given by shaded areas. (C) Histogram of the first time $b_{01}$ increases in frequency above $b_{10}$, recorded from the 200 simulations. Approximately $11 \%$ of simulations did not have an intersection between $y_{b 01}$ and $y_{b 10}$ during the simulated 50 y (marked in the histogram by the $>50$ bar). ( $D$ and $E$ ) Heat maps of the increase in relative frequency of $b_{01}$ following vaccination $\left(R_{0}{ }^{10}=1.485 ; R_{0}{ }^{00}=R_{0}{ }^{01}=1.5 ; R_{0}\left(a^{j}\right)=1.5 R_{0}\left(b^{j}\right) ; \gamma=0.6, \psi=0.9\right.$, and $\omega^{10}=1-\omega^{01}=0.95$ when they are not varied).

with observations of increase in antibiotic resistance frequencies within nVTs (24-32), but it is also possible for this change to occur after more than $50 \mathrm{y}$ (Fig. $1 C$; note the $>50$ label on the $x$ axis, marking binned results of simulation runs exceeding the simulation predetermined time limit). Therefore, substantial variation in the times before surges of antibiotic-resistant nVT strains postvaccination is expected even between populations experiencing similar conditions.

As might be expected, increasing $\omega_{10}$ lowers the prevaccine frequency of $b_{01}$, leading to a higher postvaccination surge (Fig. $3 D)$. The increase in $b_{01}$ is also more pronounced at higher values of ecological interference from $a_{00}(\psi)$ (Fig. $3 D$ and $E$ ), in line with our previous results. The increase in resistance among $\mathrm{nVT}$ s also depends on the strength of serotype-specific immunity $(\gamma)$, as this determines the extent to which $b_{01}$ can realize its transmission advantage (Fig. 3E).

\section{Discussion}

Widespread use of antibiotics has led to the rise of resistance in $S$. pneumoniae (49) - even though pneumococci are often not the direct target of antibiotics, as they are usually asymptomatically carried and not treated [invasive disease might be more than three orders of magnitude lower than carriage $(50,51)]$. Existing models of antibiotic resistance typically aim to define the conditions minimizing resistance emergence or spread under different antibiotic regimes (52-57). Fewer efforts have been made to study the effects of pneumococcal vaccination on the evolution and spread of antibiotic resistance $(44,58,59)$. In this study, we show that vaccination can impact antibiotic resistance frequencies by removing competition from susceptible strains, potentially leading to a surge in antibiotic resistance among nVT pneumococci. We show that this happens under relatively mild differences in $R_{0}$ between resistant and susceptible strains, in line with the mostly indirect advantage antibiotic resistance conveys to pneumococci.

An alternative hypothesis proposed by Lehtinen et al. (44) is centered on the understanding that the removal of vaccine strains permits longer durations of carriage of nVTs. Lehtinen et al. hypothesize that some resistance genes are in epistasis with genes influencing carriage duration, and thereby removal of competition between pneumococci due to vaccination can lead to an increase in antibiotic resistance among nVTs. A crucial difference between our model and that of the aforementioned work is that we include serotype-specific immunity following natural colonization, although by no means does this have to be completely sterilizing. Indeed, stable coexistence of resistant and susceptible strains in the prevaccine era is more likely to occur, in our model, under incomplete immunity. However, in the absence of serotype-specific immunity, coexistence becomes difficult to obtain.

We assume, in our model, that resistant bacteria are less likely to cocolonize individuals already colonized with susceptible bacteria. This is in line with observed fitness cost associated with antibiotic resistance in bacteria, although estimates of this cost vary substantially (37-43). Additionally, observational studies have found an increased risk of cocolonization by resistant gastrointestinal bacteria when a patient is already colonized by bacteria resistant to different classes of antibiotics, possibly indicating that resistant bacterial strains are less likely to be competitively excluded by each other than by susceptible strains. Such a risk of cocolonization was found independently of other risk factors such as antibiotic usage and patient comorbidities $(60,61)$. Finally, experimental results suggest that patients receiving fecal microbiota transplant of susceptible bacteria are less likely to be recolonized by resistant bacteria (62).

In our model, the vaccine serotype acts as a source of ecological competition between resistant and susceptible nonvaccine serotypes. Competition between pneumococcal strains may also be mediated through acquired immunity (strain-specific or strain-transcending) or resource competition $(63,64)$. We have previously proposed that the removal of a subset of the serotypes due to vaccination may lead to substantial rearrangements between serotype and metabolic genes (18). Such VIMs could impact the competition between resistant and susceptible nonvaccine serotypes, and they form an important avenue for future work in this area.

Further high-resolution epidemiological data are needed to test our hypothesis and assess its generality. Obtaining these data will require both sampling multiple cocolonizing pneumococcal strains and characterizing their antibiotic resistance profiles. Such data would enable us to interrogate the associations between cocolonizing serotypes and their respective antibiotic resistance profiles. A lack of association would indicate that interference in carriage between resistant and susceptible pneu-mococcal strains is not a significant factor in their dynamics. However, if the data show a negative association between cocarriage of serotypes and presence of specific antibiotic resistance genes, this would suggest that the cost of antibiotic resistance translates into a reduced ability to 
cocolonize hosts infected with susceptible strains. Our framework could then be used, in combination with these data, for future planning of antibiotic prescription strategies to minimize the possibility of an increase in resistance among nonvaccine serotypes. Possible preventative measures could be to switch to alternative antibiotics after vaccination or even to antibiotics that have negative epistatic interactions with the type of resistance expected to increase (65-67). Additional genetic data from these samples could help determine whether other factors work in conjunction with the hypothesis we suggest to drive changes in the postvaccination pneumococcal population. For example, differences in metabolic gene profiles between strains of nVTs before and after vaccination, derived from sequence data, can indicate that a competition for metabolic niches is also at play (18). Furthermore, estimates of differences in rates of recombination between serotypes can help predict which serotypes can acquire resistance more readily and will be prone to different resistance profiles due to changing selective pressures.

Finally, we acknowledge that vaccination is also a potential weapon against antibiotic resistance, as it can reduce the frequency of antibiotic usage by lowering rates of infection and invasive disease (68). However, while antibiotic usage remains at high levels, we need to understand and act against possible deleterious consequences of strain-targeted vaccination on the frequency of antibiotic resistance in nonvaccine serotypes as outlined by this study and others.

\section{Methods}

Model. We model the carriage of pneumococcal strains, defined by their serotypes and their antibiotic resistance profiles, in a population eventually subjected to vaccination. In our model, each strain genotype is defined by the tuple $(i, j)$, where $i$ determines serotype and $j$ determines the antibiotic resistance allele. For the simple biallelic, two-locus case, let $i \in(a, b), j \in(00,01)$. We denote the proportion of individuals currently carrying strain $i j$ by $y_{i j} ; z_{i}$ is the proportion of the population previously exposed to serotype $i$. For example, in this simple case, the proportion of individuals carrying susceptible and resistant bacteria of serotype $a$ is $y_{a 00}$ and $y_{a 01}$, respectively; the proportion of individuals previously exposed to serotype $a$ is given by $z_{a}$.

Let us first assume that (i) hosts carrying a bacterial strain ij cannot be reinfected by a strain with the same serotype $i$ and (ii) a host infected by bacteria susceptible to antibiotics can only be coinfected by susceptible strains of bacteria (these assumptions are made to describe the simplest formulation of the model and are generalized below).

The equations of the epidemiological model are given by (full derivations are presented in SI Appendix, Supplementary Information S1):

$\frac{d y_{a 01}}{d t}=\lambda_{a 01}\left(1-z_{a}-y_{b 00}\left(1-\left(z_{a}-y_{a 01}\right)\right)\right)-\sigma_{01} y_{a 01}+p \sigma_{00} y_{a s}$,

$\frac{d y_{a 00}}{d t}=\lambda_{a 00}\left(1-z_{a}\right)-\sigma_{00} y_{a 00}$

$\frac{d z_{a}}{d t}=\lambda_{a 00}\left(1-z_{a}-y_{b 01}\left(1-\left(z_{a}-y_{a 00}\right)\right)\right)+\lambda_{a 01}\left(1-z_{a}-y_{b 00}\left(1-\left(z_{a}-y_{a 01}\right)\right)\right)-\mu z_{a}$

where $\sigma_{i j}$ is the rate of clearance, equivalent to the inverse of colonization length $D_{i j ;} ; \mu$ is the host removal rate; and $\lambda_{i j}$ is the force of infection, determined by $y_{i j} \beta_{i j}$, where $\beta_{i j}$ is the transmission rate of strain $i j$. An analogous set of equations is given for bacteria of serotype $b$. The basic reproduction number for strain ij is determined by $R_{0}^{i j}=\beta_{i j} / \sigma_{i j}$. Antibiotic resistance acquisition is also possible in our framework and will be governed by the

1. World Health Organization (2012) Measuring Impact of Streptococcus pneumoniae and Haemophilus influenzae Type b Conjugate Vaccination (World Health Org, Geneva).

2. Parks T, Barrett $L$, Jones $N$ (2015) Invasive streptococcal disease: A review for clinicians. Br Med Bull 115:77-89.

3. Ventola CL (2015) The antibiotic resistance crisis: Part 1: Causes and threats. $P \& T 40$ : 277-283.

4. Reynolds CA, Finkelstein JA, Ray GT, Moore MR, Huang SS (2014) Attributable healthcare utilization and cost of pneumonia due to drug-resistant streptococcus pneumonia: A cost analysis. Antimicrob Resist Infect Control 3:16. parameter $p$, which determines the fraction of patients acquiring antibioticresistant pneumococci instead of being cleared. When more than one locus determining antibiotic resistance is modeled, we will denote by $\omega^{l}$ the probability of acquiring resistance to a certain profile $/$ by the susceptible strain. When not varied, parameter values are set to $R_{0}^{00}=1.5, \sigma=1 / 30 \mathrm{~d}^{-1}$, and $\mu=1 / 5 \cdot 365 \mathrm{~d}^{-1}$, in accordance with pediatric pneumococcal colonization (58); $p=0.005$ for resistance acquisition scenarios (69).

To relax the two assumptions introduced above, we introduce two parameters: We represent serotype-specific immunity using a parameter $0 \leq \gamma \leq 1$, where $\gamma=1$ is equivalent to the assumption postulated above with complete specific immunity, and $\gamma=0$ corresponds to no serotypespecific immunity.

Similarly, we introduce the parameter $0 \leq \psi \leq 1$ to represent the probability that an individual carrying a susceptible strain of pneumococci will suppress cocolonization by a resistant strain due to the fitness cost of antibiotic resistance.

Adding these parameters yields the new set of equations,

$$
\frac{d y_{a 01}}{d t}=\lambda_{a 01}\left(1-y_{a 01}-\gamma\left(z_{a}-y_{a 01}\right)-\psi y_{b 00}\left(1-\left(z_{a}-y_{a 01}\right)\right)\right)-\sigma_{01} y_{a 01}+p \sigma_{00} y_{a 00}
$$

$$
\begin{aligned}
\frac{d y_{a 00}}{d t}= & \lambda_{a 00}\left(1-y_{a 00}-\gamma\left(z_{a}-y_{a 00}\right)\right)-\sigma_{00} y_{a 00} \\
\frac{d z_{a}}{d t}= & \lambda_{a 00}\left(1-y_{a 00}-\gamma\left(z_{a}-y_{a 00}\right)\right)+\lambda_{a 01} \lambda_{a 01}\left(1-y_{a 01}-\gamma\left(z_{a}-y_{a 01}\right)\right. \\
& \left.-\psi y_{b 00}\left(1-\left(z_{a}-y_{a 01}\right)\right)\right)-\mu z_{a} .
\end{aligned}
$$

We can extend this model to any number of antigenic alleles and any number of biallelic resistance loci (see SI Appendix, Supplementary Information S1). Vaccination is added to the model by reducing the $R_{0}$ of vaccine strains by $90 \%$.

Stochastic Implementation. We developed a semiindividual-based implementation of our equations based on the Gillespie stochastic simulation algorithm (SSA) implemented with the direct method (70). Variables representing colonized hosts $\left(y_{i j}\right)$ are explicitly modeled under the SSA framework, whereas previously colonized individuals are approximated via a deterministic approach: At each newly drawn time point $t_{n+1}$, the individuals previously colonized with strain $i$ are given by $z_{i}^{t_{n+1}}=\left(t_{n+1}-t_{n}\right) F_{i}\left(\vec{y}^{t_{n}}, \vec{z}^{t_{n}}, \vec{\theta}\right)+z_{i}^{t_{n}}$, where $F_{i}$ marks the differential equation defined for the deterministic dynamics of patients previously infected with $i\left(d z_{i} / d t\right) ; \vec{y}^{t_{n}}$ and $\vec{z}^{t_{n}}$ represent all values of currently and previously colonized individuals at time $t_{n}$; and $\vec{\theta}$ represents all parameters defined in the deterministic model. Since the number of previously colonized patients is much greater than the number of those currently colonized, it can be approximated by a deterministic equation (updated by the random processes). All simulations were performed with a population size of 100,000 . Finally, we constrained the number of hosts colonized by any of the different strains to be $\geq 1$, to avoid the absorbing states of strain extinction.

BAPS. Two data sets of pneumococcal genomes, collected from the United States (16) and the United Kingdom (35), were annotated using the BIGSdb software and assigned alleles with the Genome Comparator tool (with ATCC 700669 pneumococcal strain as the reference genome) (71). We examined 34 loci associated with antibiotic resistance (given in SI Appendix, Table S1) and performed an admixture analysis on predefined clusters based on serotypes in the BAPS 6 software $(72,73)$. Only serotypes containing 15 or more samples were used, leaving us with $n=514$ and $n=391$ observations for the US and UK data, respectively. Parameters used in the software were set according to the higher accuracy recommendations given in the BAPS manual: 50 max clusters, 500 iterations, 200 reference individuals, and 20 iterations per reference individuals. The admixture inclusion threshold was set to a maximum $P$ value of 0.001 .

5. Einarsson S, Kristjansson M, Kristinsson KG, Kjartansson G, Jonsson S (1998) Pneumonia caused by penicillin-non-susceptible and penicillin-susceptible pneumococci in adults: A case-control study. Scand J Infect Dis 30:253-256.

6. Schrag SJ, et al. (2001) Resistant Pneumococcal Infections: The Burden of Disease and Challenges in Monitoring and Controlling Antimicrobial Resistance (World Health Org, Geneva).

7. Kim L, McGee L, Tomczyk S, Beall B (2016) Biological and epidemiological features of antibiotic-resistant Streptococcus pneumoniae in pre-and post-conjugate vaccine eras: A United States perspective. Clin Microbiol Rev 29:525-552.

8. Metlay JP, et al. (2000) Impact of penicillin susceptibility on medical outcomes for adult patients with bacteremic pneumococcal pneumonia. Clin Infect Dis 30:520-528. 
9. Feikin DR, et al. (2000) Mortality from invasive pneumococcal pneumonia in the era of antibiotic resistance, 1995-1997. Am J Public Health 90:223-229.

10. Tleyjeh IM, Tlaygeh HM, Hejal R, Montori VM, Baddour LM (2006) The impact of penicillin resistance on short-term mortality in hospitalized adults with pneumococcal pneumonia: A systematic review and meta-analysis. Clin Infect Dis 42:788-797.

11. Brown J, Hammerschmidt S, Orihuela C (2015) Streptococcus Pneumoniae: Molecular Mechanisms of Host-Pathogen Interactions (Academic, New York).

12. Hanage WP (2007) Serotype replacement in invasive pneumococcal disease: Where do we go from here? J Infect Dis 196:1282-1284.

13. Weinberger DM, Malley R, Lipsitch M (2011) Serotype replacement in disease after pneumococcal vaccination. Lancet 378:1962-1973.

14. Lipsitch M (1999) Bacterial vaccines and serotype replacement: Lessons from Haemophilus influenzae and prospects for Streptococcus pneumoniae. Emerg Infect Dis 5:336-345.

15. Beall BW, et al. (2011) Shifting genetic structure of invasive serotype 19A pneumococci in the United States. J Infect Dis 203:1360-1368.

16. Croucher NJ, et al. (2013) Population genomics of post-vaccine changes in pneumococcal epidemiology. Nat Genet 45:656-663.

17. Gupta S, Ferguson NM, Anderson RM (1997) Vaccination and the population structure of antigenically diverse pathogens that exchange genetic material. Proc Biol Sci 264 1435-1443.

18. Watkins ER, et al. (2015) Vaccination drives changes in metabolic and virulence profiles of Streptococcus pneumoniae. PLoS Pathog 11:e1005034.

19. Dagan R, Muallem M, Melamed R, Leroy O, Yagupsky P (1997) Reduction of pneumococcal nasopharyngeal carriage in early infancy after immunization with tetravalent pneumococcal vaccines conjugated to either tetanus toxoid or diphtheria toxoid. Pediatr Infect Dis J 16:1060-1064.

20. Dagan R, Givon-Lavi N, Zamir O, Fraser D (2003) Effect of a nonavalent conjugate vaccine on carriage of antibiotic-resistant Streptococcus pneumoniae in day-care centers. Pediatr Infect Dis J 22:532-540.

21. Mbelle N, et al. (1999) Immunogenicity and impact on nasopharyngeal carriage of a nonavalent pneumococcal conjugate vaccine. J Infect Dis 180:1171-1176.

22. Mishra RP, Oviedo-Orta E, Prachi P, Rappuoli R, Bagnoli F (2012) Vaccines and antibiotic resistance. Curr Opin Microbiol 15:596-602.

23. Chiba N, et al.; Invasive Pneumococcal Diseases Surveillance Study Group (2013) Rapid decrease of 7-valent conjugate vaccine coverage for invasive pneumococcal disease in pediatric patients in Japan. Microb Drug Resist 19:308-315.

24. Moore MR, et al. (2008) Population snapshot of emergent Streptococcus pneumoniae serotype 19A in the United States, 2005. J Infect Dis 197:1016-1027.

25. Hanage WP, et al. (2007) Diversity and antibiotic resistance among nonvaccine se rotypes of Streptococcus pneumoniae carriage isolates in the post-heptavalent conjugate vaccine era. J Infect Dis 195:347-352.

26. Kyaw MH, et al.; Active Bacterial Core Surveillance of the Emerging Infections Program Network (2006) Effect of introduction of the pneumococcal conjugate vaccine on drug-resistant Streptococcus pneumoniae. N Engl J Med 354:1455-1463.

27. Park SY, et al. (2008) Impact of conjugate vaccine on transmission of antimicrobialresistant Streptococcus pneumoniae among Alaskan children. Pediatr Infect Dis J 27 335-340.

28. Huang SS, et al. (2005) Post-PCV7 changes in colonizing pneumococcal serotypes in 16 Massachusetts communities, 2001 and 2004. Pediatrics 116:e408-e413.

29. Pillai DR, et al. (2009) Genome-wide dissection of globally emergent multi-drug re sistant serotype 19A Streptococcus pneumoniae. BMC Genomics 10:642.

30. Gherardi G, et al. (2012) Serotype and clonal evolution of penicillin-nonsusceptible invasive Streptococcus pneumoniae in the 7-valent pneumococcal conjugate vaccine era in Italy. Antimicrob Agents Chemother 56:4965-4968.

31. Dagan R, Klugman KP (2008) Impact of conjugate pneumococcal vaccines on antibiotic resistance. Lancet Infect Dis 8:785-795.

32. Farrell DJ, Klugman KP, Pichichero M (2007) Increased antimicrobial resistance among nonvaccine serotypes of Streptococcus pneumoniae in the pediatric population afte the introduction of 7-valent pneumococcal vaccine in the United States. Pediatr Infect Dis J 26:123-128.

33. Greene SK, et al. (2012) Trends in antibiotic use in Massachusetts children, 2000-2009. Pediatrics 130:15-22.

34. Grijalva CG, Nuorti JP, Griffin MR (2009) Antibiotic prescription rates for acute re spiratory tract infections in US ambulatory settings. JAMA 302:758-766.

35. Gladstone RA, et al. (2015) Five winters of pneumococcal serotype replacement in UK carriage following PCV introduction. Vaccine 33:2015-2021.

36. Diekmann O, Heesterbeek JAP (2000) Mathematical Epidemiology of Infectious Diseases: Model Building, Analysis and Interpretation (Wiley, New York), Vol 5.

37. Maher MC, et al. (2012) The fitness cost of antibiotic resistance in Streptococcus pneumoniae: Insight from the field. PLoS One 7:e29407.

38. Rozen DE, McGee L, Levin BR, Klugman KP (2007) Fitness costs of fluoroquinolone resistance in Streptococcus pneumoniae. Antimicrob Agents Chemother 51:412-416.

39. Trzciński K, Thompson CM, Gilbey AM, Dowson CG, Lipsitch M (2006) Incremental increase in fitness cost with increased $\beta$-lactam resistance in pneumococci evaluated by competition in an infant rat nasal colonization model. $J$ Infect Dis 193:1296-1303.

40. Albarracín Orio AG, Piñas GE, Cortes PR, Cian MB, Echenique J (2011) Compensatory evolution of pbp mutations restores the fitness cost imposed by $\beta$-lactam resistance in Streptococcus pneumoniae. PLoS Pathog 7:e1002000.

41. Andersson DI, Hughes D (2010) Antibiotic resistance and its cost: Is it possible to reverse resistance? Nat Rev Microbiol 8:260-271.
42. Andersson DI, Levin BR (1999) The biological cost of antibiotic resistance. Curr Opin Microbiol 2:489-493.

43. Lenski RE (1998) Bacterial evolution and the cost of antibiotic resistance. Int Microbiol $1: 265-270$

44. Lehtinen S, et al. (2017) Evolution of antibiotic resistance is linked to any genetic mechanism affecting bacterial duration of carriage. Proc Natl Acad Sci USA 114: 1075-1080.

45. Gupta S, Swinton J, Anderson RM (1994) Theoretical studies of the effects of heterogeneity in the parasite population on the transmission dynamics of malaria. Proc Biol Sci 256:231-238.

46. Lipsitch M, Bergstrom CT, Levin BR (2000) The epidemiology of antibiotic resistance in hospitals: Paradoxes and prescriptions. Proc Natl Acad Sci USA 97:1938-1943.

47. Colijn C, et al. (2009) What is the mechanism for persistent coexistence of drugsusceptible and drug-resistant strains of Streptococcus pneumoniae? J $R$ Soc Interface 7 : 905-919.

48. Lipsitch M, et al. (2012) Estimating rates of carriage acquisition and clearance and competitive ability for pneumococcal serotypes in Kenya with a Markov transition model. Epidemiology 23:510-519.

49. Bell BG, Schellevis F, Stobberingh E, Goossens H, Pringle M (2014) A systematic review and meta-analysis of the effects of antibiotic consumption on antibiotic resistance. BMC Infect Dis 14:13.

50. Flasche $S$, et al. (2011) Effect of pneumococcal conjugate vaccination on serotypespecific carriage and invasive disease in England: A cross-sectional study. PLoS Med 8: e1001017.

51. Obaro S, Adegbola R (2002) The pneumococcus: Carriage, disease and conjugate vaccines. J Med Microbiol 51:98-104.

52. Opatowski L, Guillemot D, Boëlle PY, Temime L (2011) Contribution of mathematical modeling to the fight against bacterial antibiotic resistance. Curr Opin Infect Dis 24: 279-287.

53. Caudill L, Wares JR (2016) The role of mathematical modeling in designing and evaluating antimicrobial stewardship programs. Curr Treat Options Infect Dis 8: 124-138.

54. Obolski U, Stein GY, Hadany L (2015) Antibiotic restriction might facilitate the emergence of multi-drug resistance. PLoS Comput Biol 11:e1004340.

55. Obolski U, Hadany L (2012) Implications of stress-induced genetic variation for minimizing multidrug resistance in bacteria. BMC Med 10:89.

56. Day T, Gandon S (2012) The evolutionary epidemiology of multilocus drug resistance. Evolution 66:1582-1597.

57. Bonhoeffer S, Lipsitch M, Levin BR (1997) Evaluating treatment protocols to prevent antibiotic resistance. Proc Natl Acad Sci USA 94:12106-12111.

58. Mitchell PK, Lipsitch M, Hanage WP (2015) Carriage burden, multiple colonization and antibiotic pressure promote emergence of resistant vaccine escape pneumococci. Philos Trans R Soc Lond B Biol Sci 370:20140342.

59. Temime L, Guillemot D, Boëlle PY (2004) Short- and long-term effects of pneumococcal conjugate vaccination of children on penicillin resistance. Antimicrob Agents Chemother 48:2206-2213.

60. Papadimitriou-Olivgeris $\mathrm{M}$, et al. (2015) Co-colonization by multidrug-resistant bacteria in two Greek intensive care units. Eur J Clin Microbiol Infect Dis 34:1947-1955.

61. Hayakawa K, et al. (2013) Independent risk factors for the co-colonization of vancomycinresistant Enterococcus faecalis and methicillin-resistant Staphylococcus aureus in the region most endemic for vancomycin-resistant Staphylococcus aureus isolation. Eur J Clin Microbiol Infect Dis 32:815-820.

62. Cohen NA, Maharshak N (2017) Novel indications for fecal microbial transplantation: Update and review of the literature. Dig Dis Sci 62:1131-1145.

63. Cobey S, Lipsitch M (2013) Pathogen diversity and hidden regimes of apparent competition. Am Nat 181:12-24.

64. Lourenço J, et al. (2017) Lineage structure of Streptococcus pneumoniae may be driven by immune selection on the groEL heat-shock protein. Sci Rep 7:9023.

65. Pál C, Papp B, Lázár V (2015) Collateral sensitivity of antibiotic-resistant microbes. Trends Microbiol 23:401-407.

66. Imamovic L, Sommer MO (2013) Use of collateral sensitivity networks to design drug cycling protocols that avoid resistance development. Sci Transl Med 5:204ra132.

67. Obolski U, Dellus-Gur E, Stein GY, Hadany L (2016) Antibiotic cross-resistance in the lab and resistance co-occurrence in the clinic: Discrepancies and implications in E.coli. Infect Genet Evol 40:155-161.

68. O'Neill J (2016) Infection Prevention, Control and Surveillence: Limiting the Development and Spread of Drug Resistance (Rev Antimicrobial Resistance, London), pp 1-32.

69. Fish DN, Piscitelli SC, Danziger LH (1995) Development of resistance during antimicrobial therapy: A review of antibiotic classes and patient characteristics in 173 studies. Pharmacotherapy 15:279-291.

70. Gillespie DT (1976) A general method for numerically simulating the stochastic time evolution of coupled chemical reactions. J Comput Phys 22:403-434.

71. Jolley KA, Maiden MC (2010) BIGSdb: Scalable analysis of bacterial genome variation at the population level. BMC Bioinformatics 11:595.

72. Corander J, Marttinen P (2006) Bayesian identification of admixture events using multilocus molecular markers. Mol Ecol 15:2833-2843.

73. Corander J, Marttinen P, Sirén J, Tang J (2008) Enhanced Bayesian modelling in BAPS software for learning genetic structures of populations. BMC Bioinformatics 9:539. 